\title{
The Hydration Structure of Aqueous Carbonic Acid from X-ray Absorption Spectroscopy
}

\author{
Royce K. Lam ${ }^{\mathrm{a}, \mathrm{b}}$, Alice H. England ${ }^{\mathrm{a}, \mathrm{b}, 1}$, Alex T. Sheardy ${ }^{\mathrm{a}, \mathrm{b}}$, Orion Shih ${ }^{\mathrm{a}}$, Jacob W. Smith ${ }^{\mathrm{a}, \mathrm{b}}$, \\ Anthony M. Rizzuto ${ }^{\mathrm{a}, \mathrm{b}}$, David Prendergast $^{\mathrm{c}}$, Richard J. Saykally ${ }^{* \mathrm{a}, \mathrm{b}}$
}

${ }^{a}$ Department of Chemistry, University of California, Berkeley, California 94720

${ }^{\mathrm{b}}$ Chemical Sciences Division, Lawrence Berkeley National Laboratory, Berkeley, California 94720

${ }^{c}$ Molecular Foundry, Lawrence Berkeley National Laboratory, Berkeley, California 94720

\section{Corresponding Author}

*Department of Chemistry

D31 Hildebrand Hall

University of California, Berkeley, CA 94720

Phone: 510-642-1047

Email: saykally@berkeley.edu

\section{Present Addresses}

${ }^{1}$ Oregon Health and Science University, Portland, Oregon 97207, USA 


\title{
GRAPHICAL ABSTRACT
}

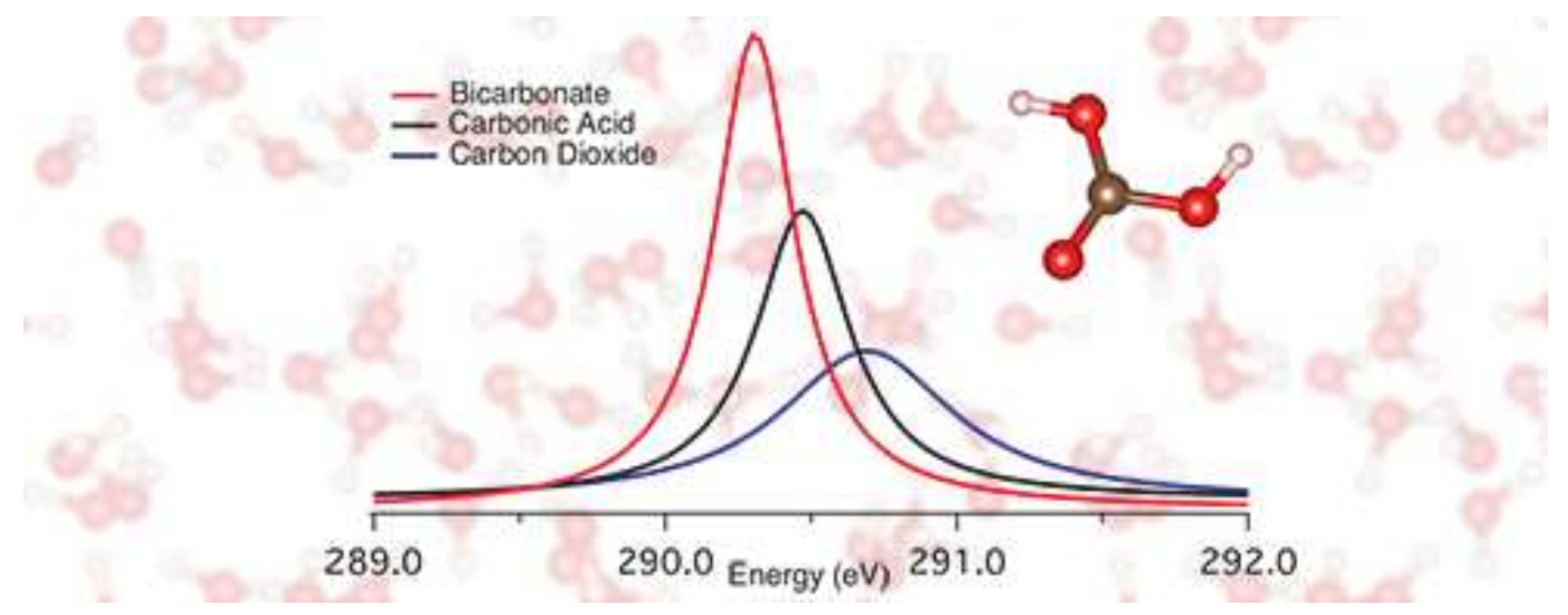

\begin{abstract}
Despite much effort, aqueous carbonic acid $\left(\mathrm{H}_{2} \mathrm{CO}_{3}\right)$ remains poorly characterized because it is very short-lived. We describe the detection and characterization of aqueous $\mathrm{H}_{2} \mathrm{CO}_{3}$ by $\mathrm{X}$-ray absorption spectroscopy, wherein protonation of a bicarbonate solution continuously generates the acid under ambient conditions. Accompanying first principles calculations of the carbon Kedge transitions facilitate spectral assignment and interpretation in terms of the $\mathrm{H}_{2} \mathrm{CO}_{3} \pi^{*}$ orbital, which exhibits a small $(0.2 \mathrm{eV})$, systematic blueshift relative to that of bicarbonate. These results establish the detailed hydration properties of this short-lived molecule and will thereby facilitate future studies of carbonate chemistry in biological and geological system.
\end{abstract}

KEYWORDS Bicarbonate, Carbon Cycle, Carbonates, Liquid Jet, Water 


\section{INTRODUCTION}

Aqueous carbonic acid $\left(\mathrm{H}_{2} \mathrm{CO}_{3}\right)$ is the centerpiece of both the global carbon cycle ${ }^{1-3}$ and physiological buffer and respiration systems, ${ }^{4-6}$ yet it remains incompletely characterized despite enormous effort. As described in a very recent review, ${ }^{7}$ carbonic acid has been well-studied in both the gas phase ${ }^{8-10}$ and in cryogenic matrixes,${ }^{11-15}$ but there have been very few successful spectroscopic studies of the aqueous acid, and none of its electronic structure. ${ }^{16-18}$ Carbonic acid is intrinsically unstable upon contact with even a single water molecule, reacting via a proton chain mechanism to rapidly form aqueous bicarbonate, carbonate, and hydrated protons, ${ }^{19-21}$ which comprises the reversible mechanism for dissolution of $\mathrm{CO}_{2}$ gas. Here we report the first measurement of X-ray absorption spectra for aqueous carbonic acid, enabled by the use of rapidflow liquid microjet technology, ${ }^{22-24}$ wherein protonation of a bicarbonate solution continuously generates the acid as a function of $\mathrm{pH}$ under ambient conditions. We combine molecular dynamics (MD) simulations with a first principles density functional theory (DFT) method ${ }^{25}$ to model and interpret the small $(0.2 \mathrm{eV})$ measured blueshift of the carbonic acid $\pi^{*}$ orbital K-edge spectra relative to those of bicarbonate, ${ }^{22}$ thereby gaining new and detailed insights into the nature of aqueous carbonic acid. We find that the two most stable isomers donate two strong hydrogen (H-) bonds to water oxygens and accept a stronger carbonyl $\mathrm{H}$-bond and weaker $\mathrm{H}-$ bonds at the acidic oxygens, exhibiting an average hydration number of 3.17 as torsional motions rearrange the solvation structures. Because both gas phase and solid phase carbonic acid have been well-studied, this work will facilitate the development of detailed models for the reversible gas-liquid chemistry of carbon dioxide.

Near edge X-ray absorption fine structure spectroscopy (NEXAFS), is an atom-specific probe of both the electronic structure of a molecule and its local chemical environment, making it a 
method of choice for detailed characterization of hydration interactions. Until very recently, NEXAFS studies of the carbonate system were restricted to gaseous $\mathrm{CO}_{2},{ }^{26}$ and to carbonates adsorbed to a surface ${ }^{27}$ or in various solid mineral forms ${ }^{28}$ by the difficulties then inherent in addressing liquid samples by this high vacuum technology. In 2011, we reported the first NEXAFS study of aqueous carbonate and bicarbonate ions, ${ }^{22}$ which was followed by an X-ray emission study in $2014 .^{29}$ In the present experiments, we follow an approach similar to that employed by Eberle $e$ et ${ }^{16}$ in observing Raman spectra of aqueous $\mathrm{H}_{2} \mathrm{CO}_{3}$, enabled by the slow dehydration of carbonic acid $\left(k=26.3 \mathrm{~s}^{-1}, \mathrm{t}_{1 / 2}=26 \mathrm{~ms}\right)$ relative to the very rapid protonation rate of the bicarbonate anion $\left(k_{o n}=4.7 \times 10^{10} \mathrm{M}^{-1} \mathrm{~s}^{-1}, t_{1 / 2}=21.3 \mathrm{ps}\right),{ }^{17,30}$ which facilitates accumulation of carbonic acid. We combine a $0.5 \mathrm{M}$ solution of $\mathrm{NaHCO}_{3}$ with $1 \mathrm{M} \mathrm{HCl}$ in a high velocity liquid microjet mixing system to generate the acid at $25^{\circ} \mathrm{C}$.

\section{MATERIALS AND METHODS}

\section{A. Sample Preparation}

Samples $\left(0.5 \mathrm{M} \mathrm{NaHCO}_{3}\right.$ and $\left.1 \mathrm{M} \mathrm{HCl}\right)$ were prepared using $18.2 \mathrm{M} \Omega \square \mathrm{cm}$ resistivity water was obtained from a Millipore purification system. Concentrated $\mathrm{HCl}(12.1 \mathrm{M})$ was obtained from J. T. Baker. $\mathrm{NaHCO}_{3}$ with stated purity of at least $99.7 \%$ was commercially obtained from Sigma-Aldrich. Samples were used without further purification.

\section{B. Experimental Design}

Carbon K-edge total electron yield (TEY) spectra were collected at Beamline 8.0.1. A detailed description of the experimental setup has been published previously. ${ }^{31}$ Briefly, an intense $\left(>10^{11}\right.$ photons/s), high resolution $(\mathrm{E} / \Delta \mathrm{E}=7000)$, tunable soft $\mathrm{X}$-ray beam is generated from an undulator at the ALS. The beam is focused (100 x $35 \mu \mathrm{m}$ spot size) onto the liquid microjet. A 
for aqueous carbonate and carbonic acid. The simulation box for the aqueous species contained 90 TIP3P water molecules. From the generated trajectories, molecular configurations were taken from 100 uncorrelated snapshots and used in the first principles spectral calculation.

X-ray absorption spectra were calculated using the eXited state Core Hole (XCH) density field theory (DFT) method. ${ }^{25}$ In this approach, the lowest core-hole excited state is treated explicitly. Higher excited states are generated from the resulting self-consistent field. The electronic structure was calculated using PWSCF from the Quantum ESPRESSO package ${ }^{33}$ and the exchange correlation energy was calculated with the PBE exchange correlation functional under the generalized gradient approximation. ${ }^{34} \mathrm{~A}$ plane wave basis set with a 25 Ry kinetic energy cutoff and periodic boundary conditions was used to model the localized and delocalized states. The calculated spectra were then aligned relative to $\mathrm{C}(1 \mathrm{~s}) \rightarrow \pi^{*}$ peak in the experimental gaseous carbon dioxide spectrum. Isosurfaces were calculated with Quantum-ESPRESSO and rendered in VESTA. ${ }^{35}$

\section{RESULTS}

Using the fast-flow microjet mixing system, X-ray absorption spectra of bicarbonate, 1:2, 1:1, and 2:1 mole fraction acid:bicarbonate mixtures were measured. The spectrum of 1:1 acid:bicarbonate was also measured without the high-flow mixing system, wherein the increased mixing time ensured that only gaseous carbon dioxide was present at the time of measurement. Good agreement between experimentally acquired spectra with the previously calculated ${ }^{22}$ first principles eXcited state Core Hole $(\mathrm{XCH})$ spectra $^{25}$ establish the predictive nature of the $\mathrm{XCH}$ method and indicate that aqueous carbonic acid is indeed the short-lived intermediate in the hydrolysis of carbon dioxide to form carbonate. Details of the hydration structure of the acid are revealed in the molecular dynamics simulations underlying the calculated $\mathrm{XCH}$ spectra. ${ }^{22}$ 
The NEXAFS data are shown in Figure 1a. Spectra have been peak-normalized and offset for clarity. All of these experimental spectra, with the exception of that for gaseous carbon dioxide, exhibit a broad resonance between 293 and $305 \mathrm{eV}$. Through comparison with the calculated isosurfaces of excited electronic states in this energy range, this resonance is assigned to a $\mathrm{C}(1 \mathrm{~s})$ $\rightarrow \sigma^{*}$ transition for either bicarbonate or carbonic acid, depending on $\mathrm{pH}$. The sharp peaks centered at $\sim 288 \mathrm{eV}$ (Fig 1b), are assigned to the respective $\mathrm{C}(1 \mathrm{~s}) \rightarrow \pi^{*}$ transitions for the different carbonate species. Isosurfaces for the $\pi^{*}(\mathrm{C}=\mathrm{O})$ state are shown in Figure 2 for bicarbonate (2a), carbonic acid (2b), dissolved carbon dioxide (2c), and gaseous carbon dioxide (2d). These images were chosen from molecular configurations whose spectra closely matched the simulated average spectra of the thermodynamic ensembles. The experimental $\mathrm{C}(1 \mathrm{~s}) \rightarrow \pi^{*}$ peaks have been fit to a Lorentzian distribution (Fig. 3) to facilitate the comparison with the calculated spectra. In the $\mathrm{C}(1 \mathrm{~s}) \rightarrow \pi^{*}$ transition, a reproducible blue shift $(\sim 0.2 \mathrm{eV})$ from the bicarbonate spectrum to the 1:1 acid:bicarbonate mixture is observed. Furthermore, the gaseous carbon dioxide (1:1 mixture without the mixing system) spectrum exhibits an additional blue shift $(\sim 0.2 \mathrm{eV})$ relative to the 1:1 mixture acquired using the fast-flow microjet mixing system. For the 1:2 acid:bicarbonate mixture, a broader $\mathrm{C}(1 \mathrm{~s}) \rightarrow \pi^{*}$ peak is observed. This feature spans the width of the peaks observed in bicarbonate and in the 1:1 mixture, indicating that both bicarbonate and the species in present in the 1:1 mixture were also present in the 1:2 mixture. The 2:1 acid:bicarbonate mixture produced the same shift as the 1:1 mixture.

To assign the measured spectral features to specific species, the experimentally measured spectra were compared to our $^{22}$ previously-calculated spectra (Fig. 4). The theoretical spectra were energy-aligned relative to the $\mathrm{C}(1 \mathrm{~s}) \rightarrow \pi^{*}$ transition for gaseous carbon dioxide. Comparison of the $\sigma^{*}$ features confirmed that the species absorbing in the $1: 1$ and $2: 1$ 
respective hydration environments. It is likely that this slightly altered hydrogen bonding structure results from the different electronic structure methods used.

\section{CONCLUSIONS}

In summary, this first characterization of carbonic acid under ambient aqueous conditions by the atom selective technique of $\mathrm{x}$-ray absorption spectroscopy and first principles $\mathrm{XCH}$ calculations establishes the detailed hydration properties of this short-lived species. Calculated spectral energy shifts and intensities for the species observed (bicarbonate, carbonic acid, gaseous carbon dioxide) correspond well with the experimentally measured spectra, demonstrating the predictive power of the $\mathrm{XCH}$ technique for calculating reliable core-level spectra. We expect that these results will thereby facilitate future studies of the carbon dioxide chemistry in geological and biological systems.

\section{ACKNOWLEDGMENTS}

This work was supported by the Director, Office of Basic Energy Sciences, Office of Science, U.S. Department of Energy (DOE) under Contract No. DE-AC02-05CH11231, through LBNL Chemical Sciences Division. Computational resources were provided by the National Energy Research Scientific Computing Center (NERSC), a DOE Advanced Scientific Computing Research User Facility. The authors thank Wanli Yang and Jon Spear for beamline support at the Advanced Light Source. The data presented are available upon request to saykally@berkeley.edu.

\section{REFERENCES}

(1) Putnis, A. Why Mineral Interfaces Matter. Science 2014, 343, 1441-1442.

(2) Al-Hosney, H. A.; Grassian, V. H. Carbonic Acid: An Important Intermediate in the Surface Chemistry of Calcium Carbonate. J. Am. Chem. Soc. 2004, 126, 8068-8069. 
(3) Ridgwell, A.; Zeebe, R. E. The Role of the Global Carbonate Cycle in the Regulation and Evolution of the Earth System. Earth Planet. Sci. Lett. 2005, 234, 299-315.

(4) Silverman, D. N.; McKenna, R. Solvent-Mediated Proton Transfer in Catalysis by Carbonic Anhydrase. Acc. Chem. Res. 2007, 40, 669-675.

(5) Kurtz, I.; Kraut, J.; Ornekian, V.; Nguyen, M. K. Acid-Base Analysis: A Critique of the Stewart and Bicarbonate-Centered Approaches. Am. J. Physiol. - Ren. Physiol. 2008, 294, F1009-F1031.

(6) Silverman, D. N.; Lindskog, S. The Catalytic Mechanism of Carbonic Anhydrase: Implications of a Rate-Limiting Protolysis of Water. Acc. Chem. Res. 1988, 21, 30-36.

(7) Reddy, S. K.; Balasubramanian, S. Carbonic Acid: Molecule, Crystal and Aqueous Solution. Chem. Commun. 2014, 50, 503-514.

(8) Terlouw, J. K.; Lebrilla, C. B.; Schwarz, H. Thermolysis of NH4HCO3-A Simple Route to the Formation of Free Carbonic Acid (H2CO3) in the Gas Phase. Angew. Chemie Int. Ed. English 1987, 26, 354-355.

(9) Mori, T.; Suma, K.; Sumiyoshi, Y.; Endo, Y. Spectroscopic Detection of Isolated Carbonic Acid. J. Chem. Phys. 2009, 130, 204308.

(10) Mori, T.; Suma, K.; Sumiyoshi, Y.; Endo, Y. Spectroscopic Detection of the Most Stable Carbonic Acid, Cis-Cis H2CO3. J. Chem. Phys. 2011, 134, 044319.

(11) Moore, M. H.; Khanna, R. K. Infrared and Mass Spectral Studies of Proton Irradiated $\mathrm{H} 2 \mathrm{O}+\mathrm{CO} 2$ Ice: Evidence for Carbonic Acid. Spectrochim. Acta Part A Mol. Spectrosc. 1991, 47, 255-262.

(12) Moore, M. H.; Khanna, R.; Donn, B. Studies of Proton Irradiated H2O + CO2 and H2O + CO Ices and Analysis of Synthesized Molecules. J. Geophys. Res. Planets 1991, 96, 17541-17545.

(13) Hage, W.; Hallbrucker, A.; Mayer, E. Carbonic Acid: Synthesis by Protonation of Bicarbonate and FTIR Spectroscopic Characterization via a New Cryogenic Technique. J. Am. Chem. Soc. 1993, 115, 8427-8431.

(14) Hage, W.; Liedl, K. R.; Hallbrucker, A.; Mayer, E. Carbonic Acid in the Gas Phase and Its Astrophysical Relevance. Science 1998, 279, 1332-1335.

Bernard, J.; Huber, R. G.; Liedl, K. R.; Grothe, H.; Loerting, T. Matrix Isolation Studies of Carbonic Acid-The Vapor Phase above the B-Polymorph. J. Am. Chem. Soc. 2013, $135,7732-7737$. 
(16) Falcke, H.; Eberle, S. H. Raman Spectroscopic Identification of Carbonic Acid. Water Res. 1990, 24, 685-688.

(17) Adamczyk, K.; Premont-Schwarz, M.; Pines, D.; Pines, E.; Nibbering, E. T. J. Real-Time Observation of Carbonic Acid Formation in Aqueous Solution. Science 2009, 326, 16901694.

(18) Wang, X.; Conway, W.; Burns, R.; McCann, N.; Maeder, M. Comprehensive Study of the Hydration and Dehydration Reactions of Carbon Dioxide in Aqueous Solution. J. Phys. Chem. A 2009, 114, 1734-1740.

(19) Yamabe, S.; Kawagishi, N. A Computational Study on the Relationship between Formation and Electrolytic Dissociation of Carbonic Acid. Theor. Chem. Acc. 2011, 130, 909-918.

(20) Gallet, G. A.; Pietrucci, F.; Andreoni, W. Bridging Static and Dynamical Descriptions of Chemical Reactions: An Ab Initio Study of $\mathrm{CO} 2$ Interacting with Water Molecules. $J$. Chem. Theory Comput. 2012, 8, 4029-4039.

(21) Galib, M.; Hanna, G. Mechanistic Insights into the Dissociation and Decomposition of Carbonic Acid in Water via the Hydroxide Route: An Ab Initio Metadynamics Study. $J$. Phys. Chem. B 2011, 115, 15024-15035.

(22) England, A. H.; Duffin, A. M.; Schwartz, C. P.; Uejio, J. S.; Prendergast, D.; Saykally, R. J. On the Hydration and Hydrolysis of Carbon Dioxide. Chem. Phys. Lett. 2011, 514, 187195.

(23) Faubel, M.; Schlemmer, S.; Toennies, J. P. A Molecular Beam Study of the Evaporation of Water from a Liquid Jet. Zeitschrift für Phys. D Atoms, Mol. Clust. 1988, 10, 269-277.

(24) Wilson, K. R.; Rude, B. S.; Catalano, T.; Schaller, R. D.; Tobin, J. G.; Co, D. T.; Saykally, R. J. X-Ray Spectroscopy of Liquid Water Microjets. J. Phys. Chem. B 2001, 105, 3346-3349.

(25) Prendergast, D.; Galli, G. X-Ray Absorption Spectra of Water from First Principles Calculations. Phys. Rev. Lett. 2006, 96, 215502.

(26) Sham, T. K.; Yang, B. X.; Kirz, J.; Tse, J. S. K-Edge near-Edge X-Ray-Absorption Fine Structure of Oxygen- and Carbon-Containing Molecules in the Gas Phase. Phys. Rev. A 1989, 40, 652-669. Ag $\{110\}$ : An X-Ray Absorption Fine-Structure Study. EPL (Europhysics Lett. 1988, 5 , 443. 
(28) Brandes, J. A.; Wirick, S.; Jacobsen, C. Carbon K-Edge Spectra of Carbonate Minerals. J. Synchrotron Radiat. 2010, 17, 676-682.

(29) Horikawa, Y.; Yoshida, A.; Takahashi, O.; Arai, H.; Tokushima, T.; Gejo, T.; Shin, S. The Electronic Structure of Carbonate Ion in Aqueous Solution Studied by Soft X-Ray Emission Spectroscopy. J. Mol. Liq. 2014, 189, 9-12.

(30) Eigen, M. Proton Transfer, Acid-Base Catalysis, and Enzymatic Hydrolysis. Part I: ELEMENTARY PROCESSES. Angew. Chemie Int. Ed. English 1964, 3, 1-19.

(31) Wilson, K. R.; Rude, B. S.; Smith, J.; Cappa, C.; Co, D. T.; Schaller, R. D.; Larsson, M.; Catalano, T.; Saykally, R. J. Investigation of Volatile Liquid Surfaces by Synchrotron XRay Spectroscopy of Liquid Microjets. Rev. Sci. Instrum. 2004, 75, 725-736.

(32) Case, D. A.; Darden, T. A.; Cheatham; Simmerling, C. L.; Wang, J.; Duke, R. E.; Luo, R.; Merz, K. M.; Pearlman, D. A.; Crowley, M.; et al. Amber 9; San Francisco, 2006.

(33) Giannozzi, P.; Baroni, S.; Bonini, N.; Calandra, M.; Car, R.; Cavazzoni, C.; Ceresoli, D.; Chiarotti, G. L.; Cococcioni, M.; Dabo, I.; et al. QUANTUM ESPRESSO: A Modular and Open-Source Software Project for Quantum Simulations of Materials. J. Phys. Condens. Matter 2009, 21, 395502.

(34) Perdew, J. P.; Burke, K.; Ernzerhof, M. Generalized Gradient Approximation Made Simple. Phys. Rev. Lett. 1996, 77, 3865-3868.

(35) Momma, K.; Izumi, F. VESTA: A Three-Dimensional Visualization System for Electronic and Structural Analysis. J. Appl. Crystallogr. 2008, 41, 653-658.

(36) Kumar, P. P.; Kalinichev, A. G.; Kirkpatrick, R. J. Hydrogen-Bonding Structure and Dynamics of Aqueous Carbonate Species from Car-Parrinello Molecular Dynamics Simulations. J. Phys. Chem. B 2008, 113, 794-802.

(37) England, A. H. X-Ray Spectroscopy and Pulse Radiolysis of Aqueous Solutions, University of California, Berkeley: Ann Arbor, 2011, p. 95.

(38) Rustad, J. R.; Nelmes, S. L.; Jackson, V. E.; Dixon, D. A. Quantum-Chemical Calculations of Carbon-Isotope Fractionation in $\mathrm{CO} 2(\mathrm{~g})$, Aqueous Carbonate Species, and Carbonate Minerals. J. Phys. Chem. A 2008, 112, 542-555.

(39) Leung, K.; Nielsen, I. M. B.; Kurtz, I. Ab Initio Molecular Dynamics Study of Carbon Dioxide and Bicarbonate Hydration and the Nucleophilic Attack of Hydroxide on CO2. J. Phys. Chem. B 2007, 111, 4453-4459. 


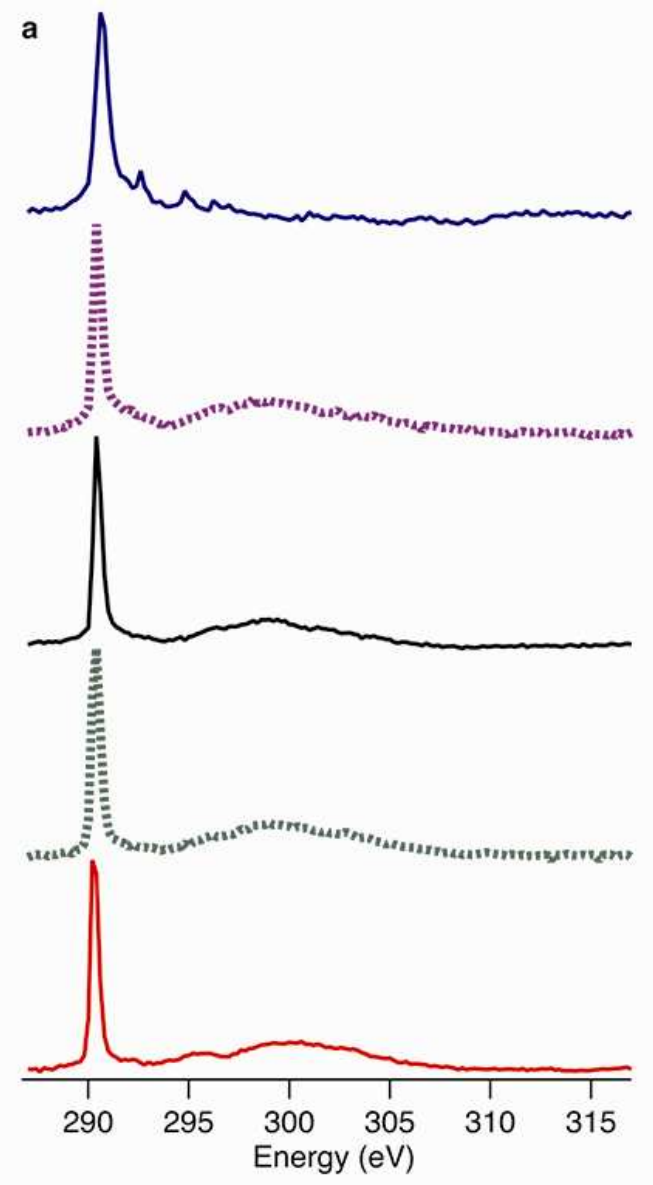

\author{
- Bicarbonate \\ "แ! 1:2 Acid:Bicarbonate \\ 1:1 Acid:Bicarbonate (Carbonic Acid) \\ …" 2:1 Acid:Bicarbonate
}

1:1 Acid:Bicarbonate (No Mixing System, Gaseous $\mathrm{CO}_{2}$ )

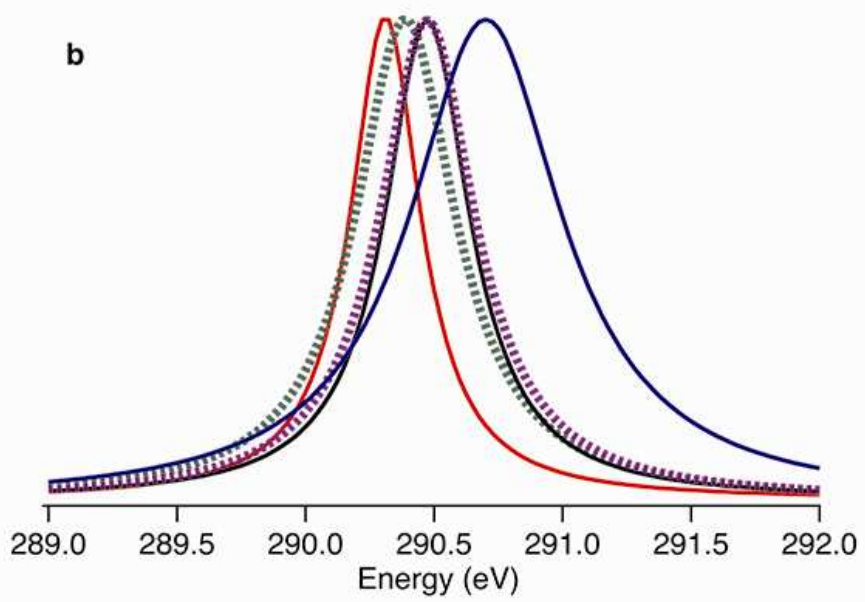

Figure 1 Peak-normalized experimental carbon K-edge X-ray absorption spectra. a. Overview of measured spectra for bicarbonate (Red), and the mixtures 1:2 $\mathrm{HCl}: \mathrm{NaHCO}_{3}$ (Green), 1:1 $\mathrm{HCl}: \mathrm{NaHCO}_{3}$ (Black), 2:1 $\mathrm{HCl}: \mathrm{NaHCO}_{3}$ (Purple), and 1:1 $\mathrm{HCl}: \mathrm{NaHCO}_{3}$ without the mixing system (Blue). b. Lorentzian fit experimental X-ray absorption spectra of the $\mathrm{C}(1 \mathrm{~s}) \rightarrow \pi^{*}$ transition in bicarbonate (Red), 1:2 $\mathrm{HCl}_{\mathrm{NaHCO}}(\mathrm{Green}), 1: 1$ $\mathrm{HCl}: \mathrm{NaHCO}_{3}$ (Black), 2:1 HCl: $\mathrm{NaHCO}_{3}$ (Purple), and 1:1 HCl: $\mathrm{NaHCO}_{3}$ without the mixing system (Blue). The maximum absorptions are at $288.31,288.41,288.49,288.49$, and $288.71 \mathrm{eV}$, respectively. All other spectra were measured using a sample introduced by the rapid-flow microjet mixing system. 


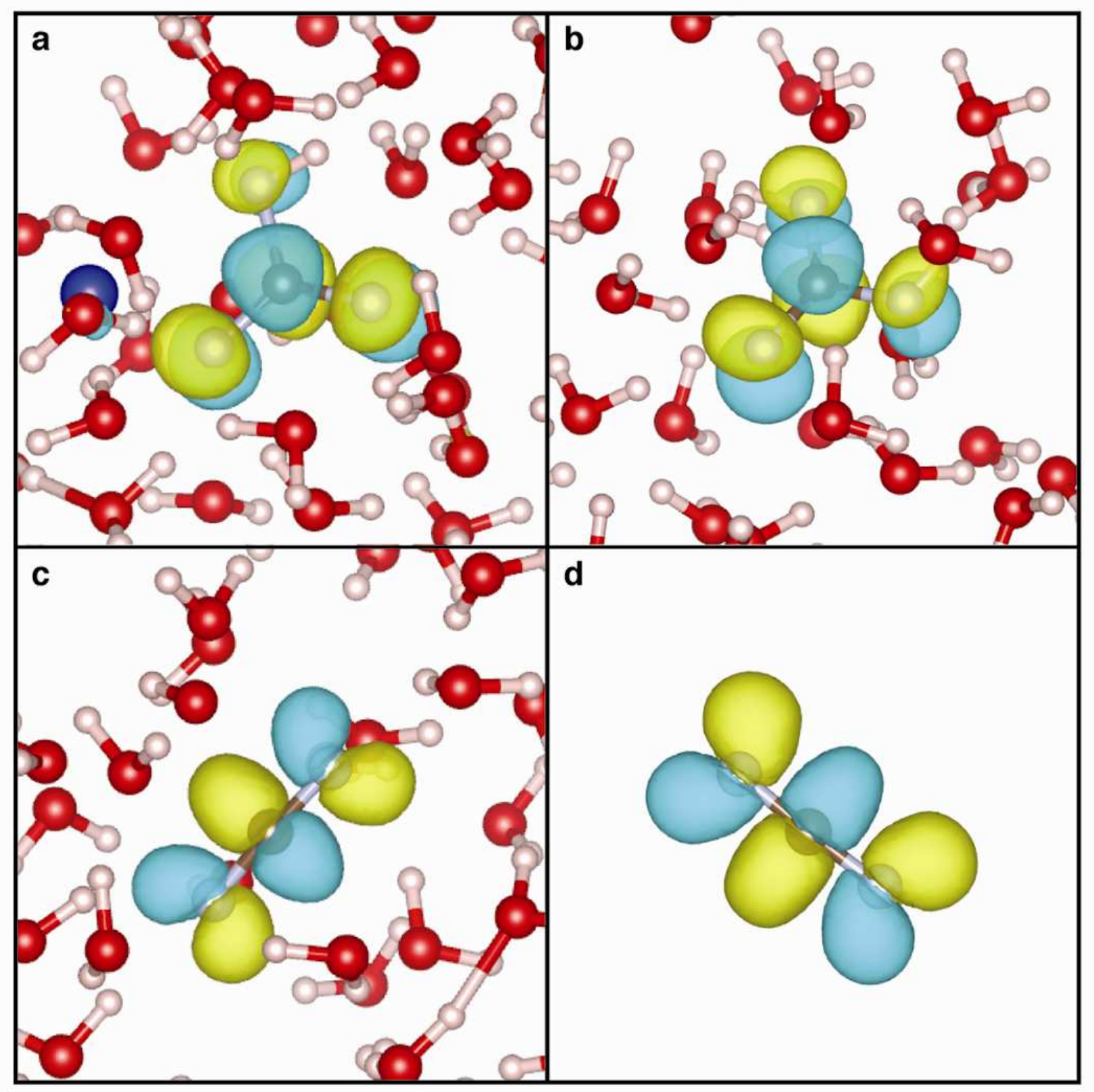

Figure 2. Isosurfaces of the core-excited electron density for $\pi^{*}$ resonances. Oxygen atoms on the carbonate species are shaded. $a . \mathrm{NaHCO}_{3} \mathrm{~b}$. $\mathrm{H}_{2} \mathrm{CO}_{3}$ c. Aqueous $\mathrm{CO}_{2}$ d. Gaseous $\mathrm{CO}_{2}$. 
Figure 3. Unnormalized experimental spectra of the $\mathrm{C}(1 \mathrm{~s}) \rightarrow \pi^{*}$ transition (solid lines) for $\mathrm{NaHCO}_{3}(\mathrm{Red}), \mathrm{H}_{2} \mathrm{CO}_{3}(\mathrm{Black})$, and $\mathrm{CO}_{2}$ (Blue). The Lorentzian fits are shown in the dashed lines. FWHM: $0.025 \mathrm{eV}\left(\mathrm{NaHCO}_{3}\right), 0.038 \mathrm{eV}\left(\mathrm{H}_{2} \mathrm{CO}_{3}\right), 0.11 \mathrm{eV}\left(\mathrm{CO}_{2(g)}\right)$. 


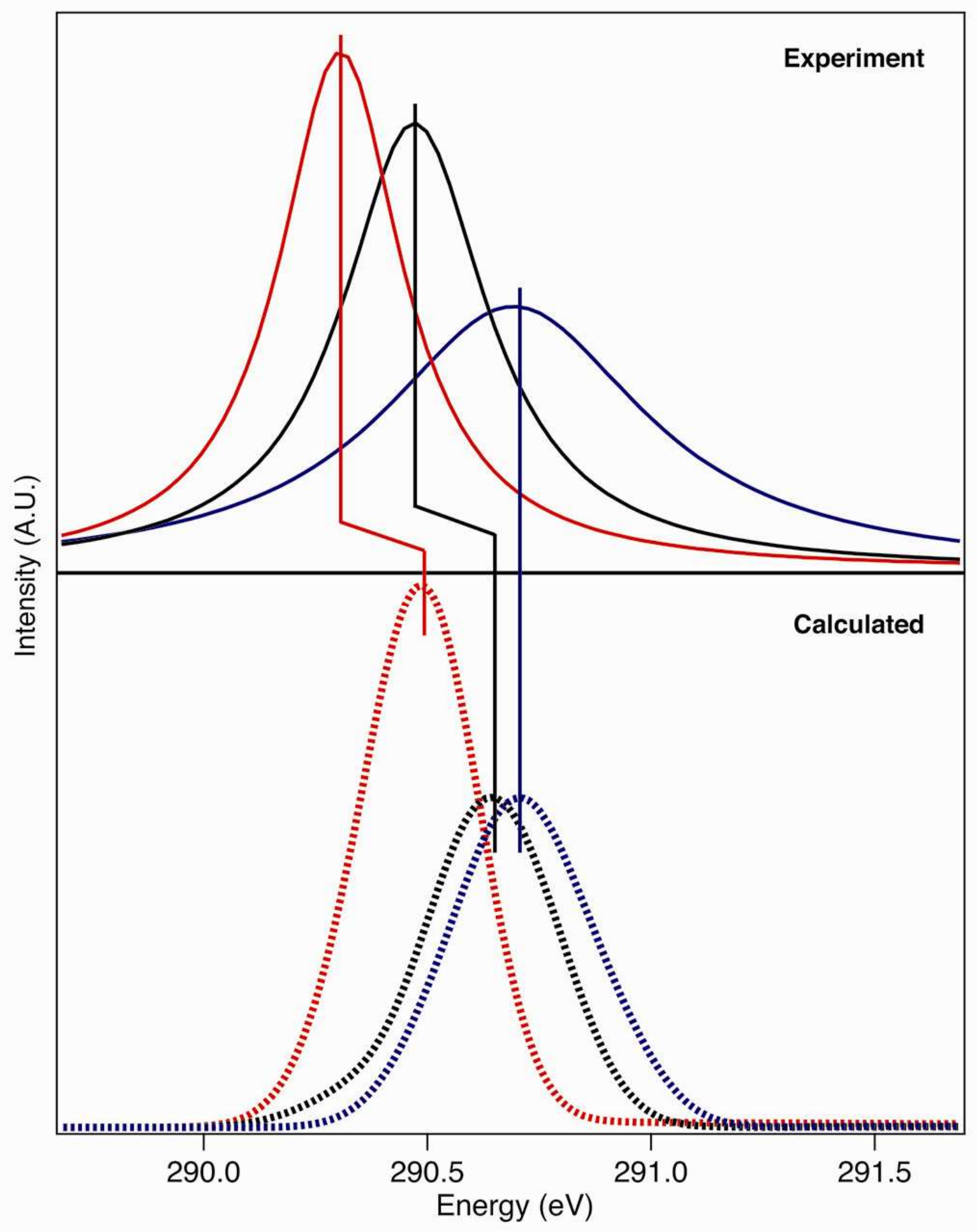

Figure 4. Lorentzian-fit experimental and calculated spectra of the $\mathrm{C}(1 \mathrm{~s}) \rightarrow \pi^{*}$ transition of carbonate species. Bicarbonate (red), carbonic acid (black), and gaseous carbon dioxide (blue). Theoretical spectra were energy-aligned to the experimental spectra for gaseous $\mathrm{CO}_{2}$. Experimental spectra are area normalized to facilitate comparison with the calculated spectra. 


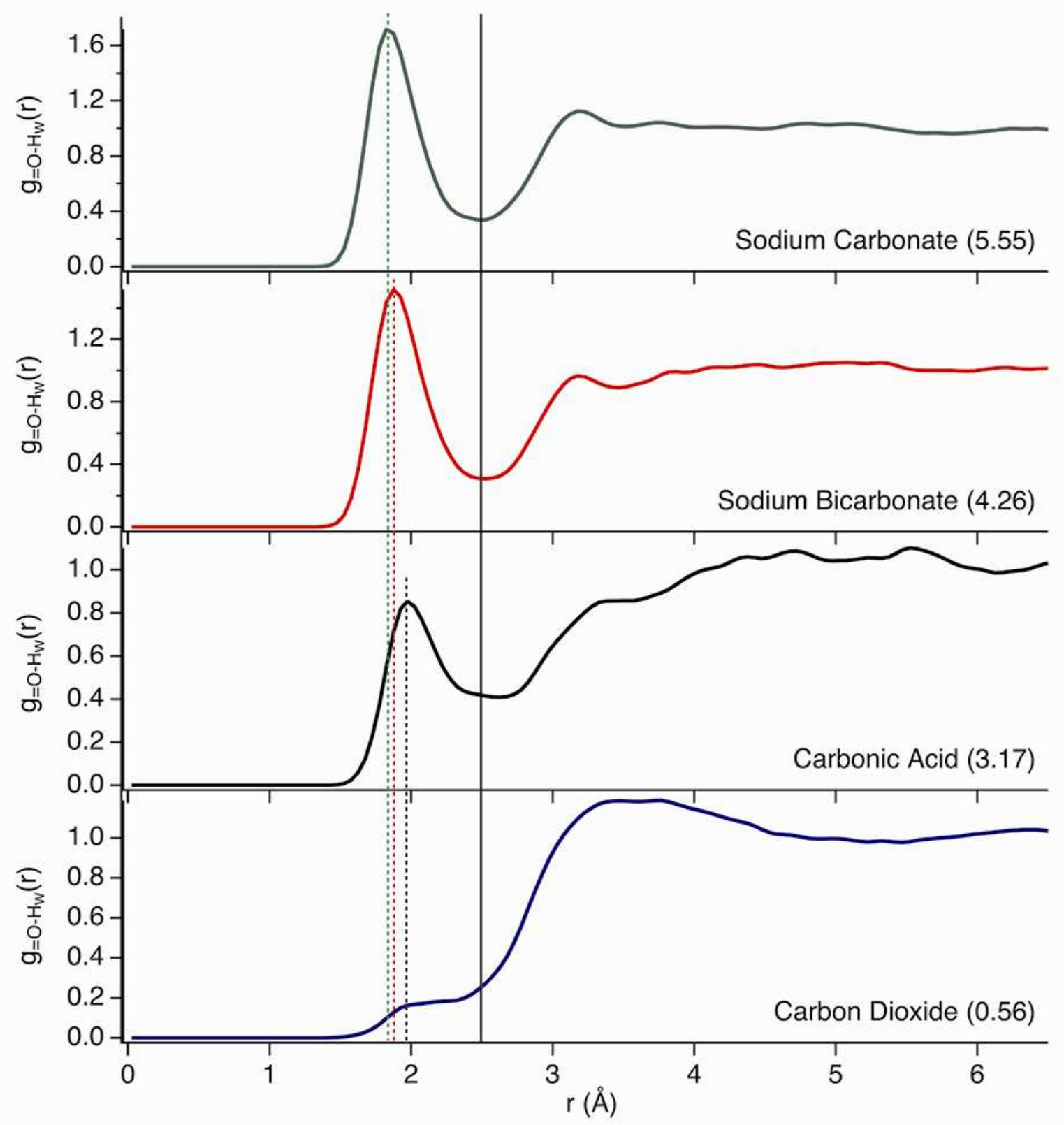

Figure 5. Calculated radial distribution functions for the carbonyl oxygen to water hydrogens of the different carbonate species. The carbonate and bicarbonate simulations include sodium counterions. Number in parentheses corresponds to the coordination number to distances of $2.5 \AA$. 


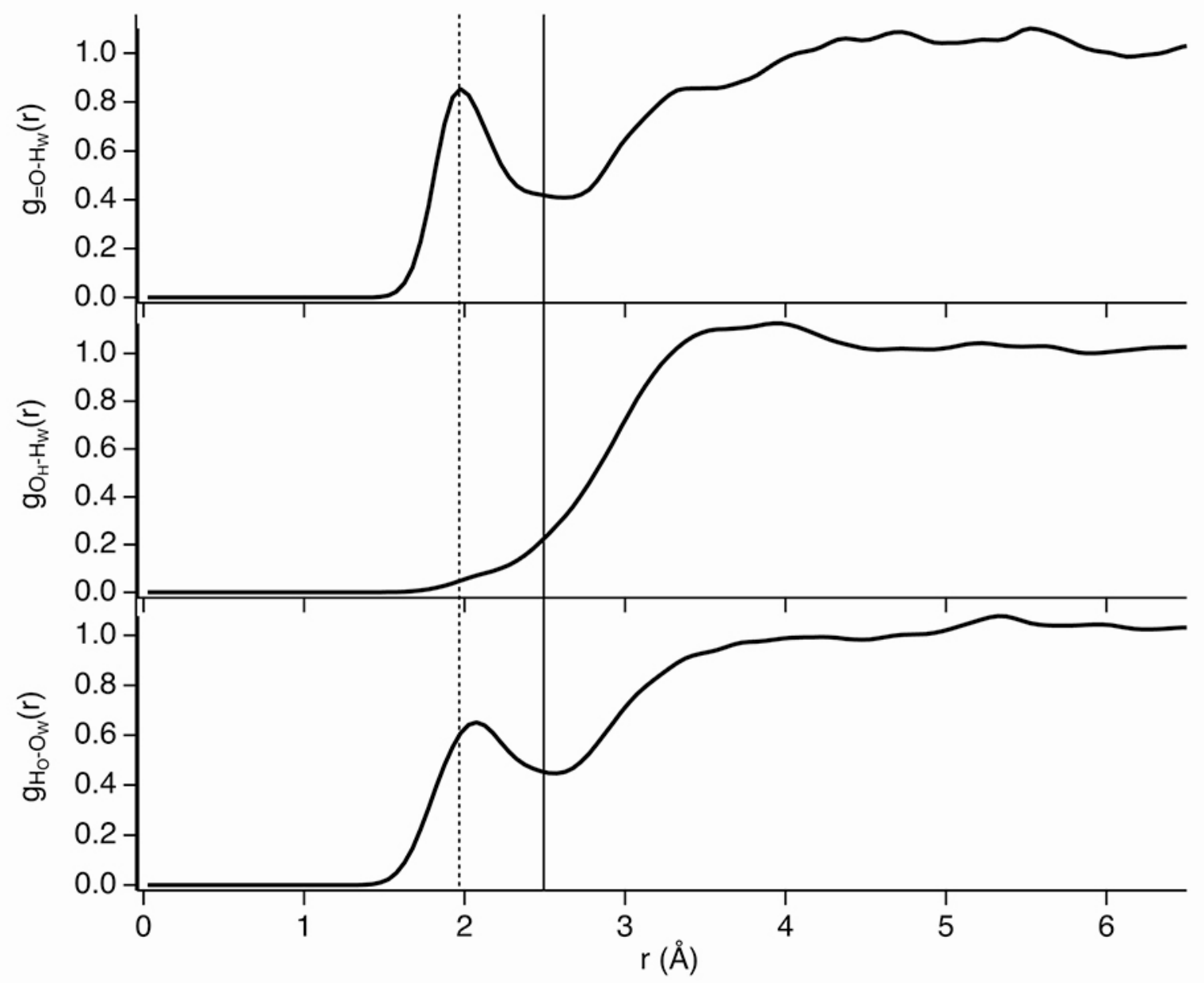

Figure 6. Calculated radial distribution functions for carbonic acid. The labels $=\mathrm{O}, \mathrm{O}_{\mathrm{H}}$, and $\mathrm{H}_{\mathrm{O}}$ refer to the carbonyl, hydroxyl oxygen, and hydroxyl hydrogen respectively. $\mathrm{O}_{\mathrm{w}}$ and $\mathrm{H}_{\mathrm{w}}$ refer to the oxygens and hydrogens of the water molecules. 


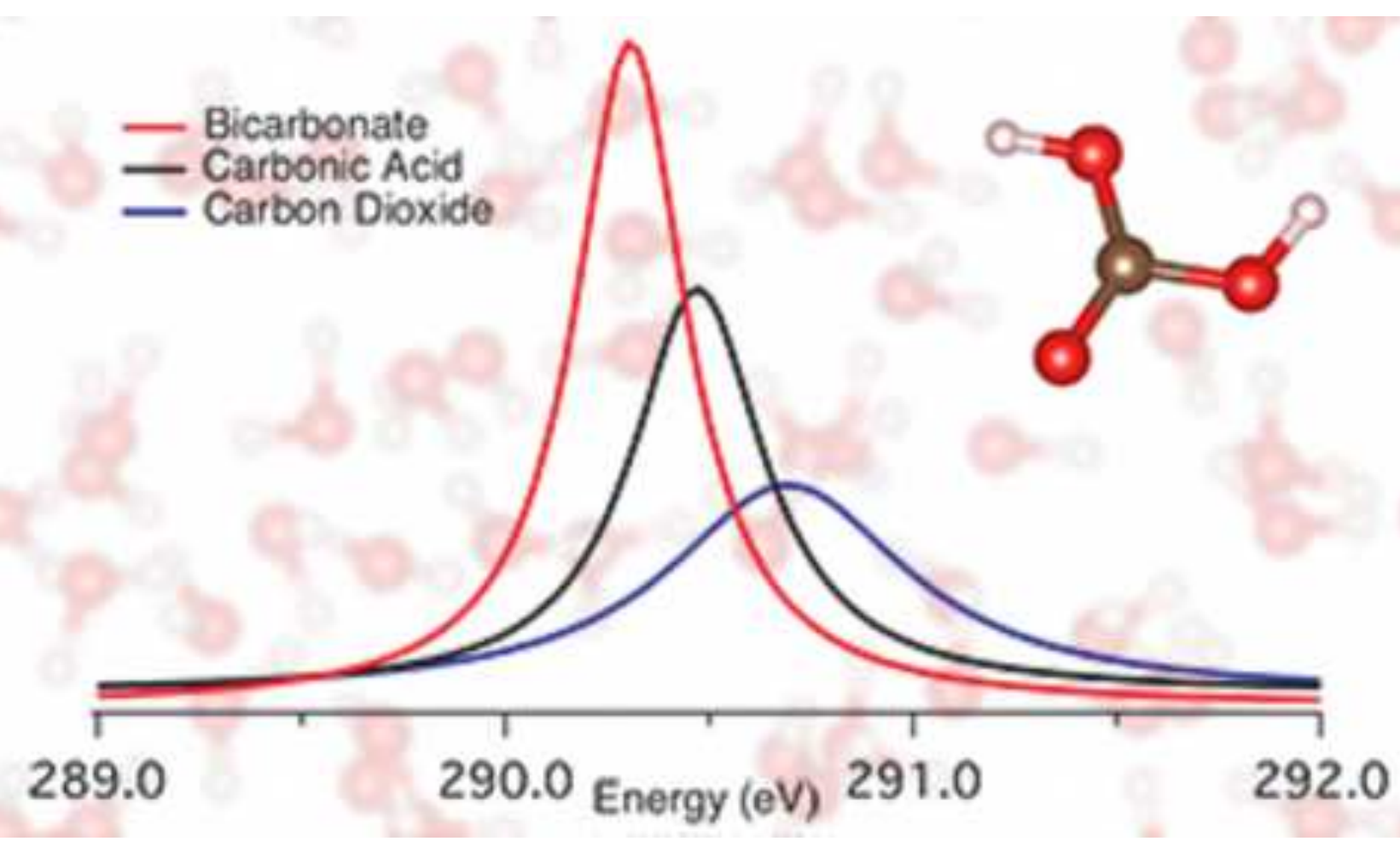

$$
290.0 \text { Energy (eV) } 291.0
$$

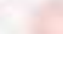

